\title{
Rendering Stimuli for SSVEP-BCI and Attention Tracking with Blinker
}

\author{
A. Chabuda $^{a}$, M. Dovgialo ${ }^{a, b}$, \\ A. DUSZYK ${ }^{c}$, J. ŻYGIEREWICZ ${ }^{a}$, \\ M. PAWLISZ ${ }^{b}$ AND P. DURKA ${ }^{a, b}$ \\ ${ }^{a}$ University of Warsaw, Faculty of Physics, L. Pasteura 5, PL-02093 Warsaw, Poland \\ ${ }^{b}$ BrainTech Ltd., Puławska 12/3, PL-02566 Warsaw, Poland \\ ${ }^{c}$ University of Social Sciences and Humanities, Faculty of Psychology, \\ Chodakowska 19/31, PL-03093 Warsaw, Poland
}

Doi: 10.12693/APhysPolA.139.426

*e-mail: anna.chabuda@fuw.edu.pl

\begin{abstract}
Brain-computer interfaces based on steady-state visual evoked potentials are currently the fastest systems for non-muscular communication, based directly on brain activity. Steady-state visual evoked potentials are evoked by a flashing light. Higher frequencies (above $30 \mathrm{~Hz}$ ), preferred for safety and comfort, cannot be reliably generated on a standard computer display. In this paper, we present and verify a complete solution, based upon a previously proposed proof of concept, which allows for highlighting an arbitrary area of the screen by LEDs flickering at strictly controlled frequencies. To validate the proposed solution in the original context of non-muscular communication, we extended our previous, general study on "brain-computer interfaces illiteracy" to include 49 subjects, which resulted in a second-largest study of high frequency steady-state visual evoked potential-based brain-computer interface. In relation to the available literature on this topic, briefly reviewed in this paper, the presented results are the most promising in terms of combined percent of successful communication among users, accuracy and the significance of estimates. It suggests that the proposed approach and device are ready for applications, opening new possibilities not only in ergonomic design of brain-computer interfaces, but also in related fields like psychophysiological or neuromarketing research involving attention control, by tracing user's attention assigned to an arbitrary area of the screen.
\end{abstract}

topics: EEG, SSVEP, BCI, Blinker

\section{Introduction}

When we look at a regularly flashing light, its frequency is reproduced in the electroencephalogram (EEG) recorded from occipital electrodes. This response is called the steady-state visual evoked potential (SSVEP) [1]. When we perceive several objects flashing simultaneously with different frequencies, the most pronounced SSVEP reflects the flashing frequency of the item we focus attention on. Seemingly, an SSVEP-based brain-computer interface (BCI) system works similarly to an eyetrackerbased technology — a user looks at the chosen button to select action, but the physiological mechanism underlying its operation is in fact entirely different, and these mechanisms cannot be treated as substitutes.

SSVEPs are the basis of the currently fastest BCIs, that is, systems providing non-muscular communication based directly on the brain activity, and offers a unique tool for tracing the attention mechanisms in physiological and psychological studies [2-7]. SSVEP responses have been measured for stimulation frequencies from 4 to $90 \mathrm{~Hz}$ [8]. In fact, the most robust SSVEP response occurs for the stimuli flashing with low $(4-12 \mathrm{~Hz})$ and mid $(12-30 \mathrm{~Hz})$ frequencies. It is easier to detect the SSVEP generated by lower frequency stimuli because, with the increase of frequency, the amplitude of SSVEP decreases. For medium and highfrequency bands, the EEG spectrum is often corrupted with broad-band muscle artifacts. However, low-frequency stimuli are unpleasant and tiring (imagine continuously looking at a broken fluorescent lamp), and in photosensitive subjects, these frequencies can trigger an epileptic seizure [9].

These problems are eliminated to a large extent when high-frequency stimuli (above $30 \mathrm{~Hz}$ ) are used. However, such stimuli cannot be efficiently rendered on a general-purpose computer screen because of the limitations of the refresh rates and nonreal-time kernels of general-use operating systems. The most common solution to this problem consists of placing external, hardware-controlled LED panels around a monitor, as in Fig. 1a. Potentially, variable screen content indicates the actions triggered 


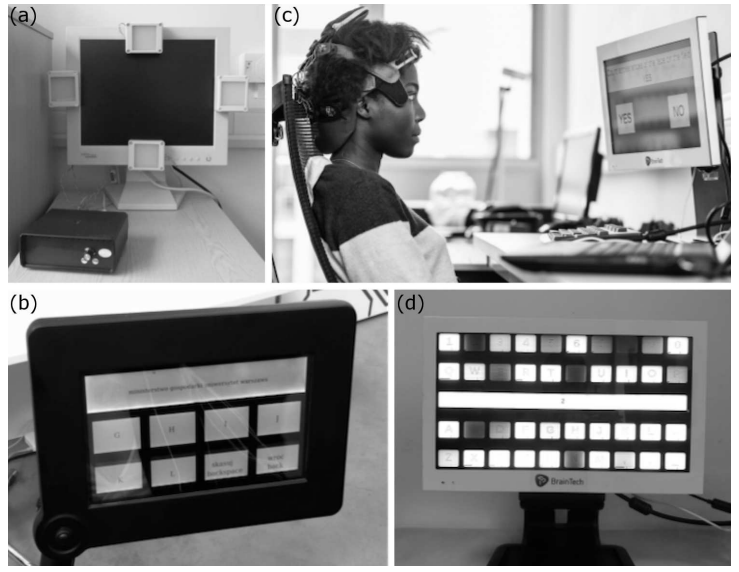

Fig. 1. (a) Classical ATM-like setup of a highfrequency S SVEP-BCI system with four fixed fields flashing outside the screen (from [10]), (b) fixed highlights architecture presented at CeBIT 2012, (c) BCI-SSVEP experiment setup based on YES/NO communication with Blinker, (d) SSVEPspeller with 40 fields with different letters and the status bar in the middle of the screen for information purposes.

by these fixed external "buttons", similarly to the classical ATMs (see Table I and [11-13, 15, 17]).

However, when compared to the buttons labeled directly with their function icons, this is not an elegant solution, so modern ATMs switch to touchscreens. In the SSVEP-BCI, this problem is even more pronounced, since we have to focus our attention first on the descriptions on the screen, and then, after making the desired choice, move the attention focus to a meaningless button to trigger the action. Also, in this setup, the number of choices in each stage is fixed. These limitations can make discussed solutions non-functional for the target users, i.e., patients with permanent paralysis. Also, the user's interaction with such a system is non-intuitive. For an efficient system applicable in real-world conditions, these aspects are just as important as advanced and robust signal processing.

In this paper, we present a solution to these problems in terms of a system capable of highlighting arbitrary areas of the screen with LEDs flashing with selected frequencies. It is based upon our previous proof of concept [10], where highlighting fields were fixed, and only information displayed in these designated areas ("buttons") could be flexibly changed by controlling the overlaid LCD. In the presented version, the topology of the areas flashing with different frequencies can be dynamically reorganized, which allows dynamically changing the shape and number of "buttons", corresponding, e.g., to BCI options labelled by letters, groups of letters, or icons. Depending on the experimental setup, SSVEPs evoked by these flashing highlights may also reveal the focus of the user's attention in psychological or neuromarketing research - future applications are yet to be explored.

\section{Blinker - hardware solution and specifications}

The main idea of Blinker (shown in Fig. 1 and first proposed in [10] as the BCI Appliance) consists of replacing standard highlights of a computer screen with an array of hardware-controlled LEDs,

\section{TABLE I}

Results of high-frequency SSVEP-BCIs operating online, including those results reported in the literature (Ref.) and the obtained in the presented experiment ("this paper"). Columns notation: \% comm - the percent of users able to communicate via BCI, \% comm (Lapl) - the percent of users applying Laplacian smoothing (3).

\begin{tabular}{c|l|c|c|c|c}
\hline \hline Ref. & \multicolumn{1}{|c|}{ Stimulator } & \# subjects & $\begin{array}{c}\text { \% mean } \\
\text { accuracy }\end{array}$ & $\begin{array}{c}\text { \% comm } \\
\text { comm } \\
(\text { Lapl })\end{array}$ \\
\hline$[11]$ & LED panels ATM-like setup & 6 & 86.6 & 100 & 87.5 \\
{$[12]$} & LED panels ATM-like setup & 15 & 95.7 & 86 & 82.4 \\
{$[13]$} & LED panels ATM-like setup & 6 & 95.5 & 100 & 87.5 \\
{$[14]$} & LED panels in a row in a maze front & 86 & 89.16 & 65.1 & 64.8 \\
{$[15]$} & LED panels ATM-like setup & 7 & 93.14 & 100 & 88.9 \\
{$[16]$} & LED-highlighted LCD & 15 & 96 & 60 & 58.8 \\
{$[17]$} & LED panels ATM-like setup & 5 & 99.2 & 100 & 85.7 \\
{$[18]$} & LCD screen with 120 Hz refresh rate & 10 & 97.75 & 100 & 91.7 \\
{$[19]$} & LED panels QWERTY board with 30 fields & 26 & 68.9 & 100 & 96.4 \\
{$[20]$} & LCD screen with 120 Hz refresh rate & 10 & 88.7 & 100 & 91.7 \\
{$[21]$} & Blinker & 28 & 93.4 & 96.4 & 93.3 \\
this paper & Blinker & 49 & 93.8 & 94.8 & 92.2
\end{tabular}


capable of flashing with individually-controlled frequencies. Previous prototypes provided 8 or 9 flashing fields with fixed geometry, determined by the highlighting elements. The frequency resolution was limited to $1 \mathrm{~Hz}$. One of these prototypes was the basis of the fastest BCI system presented at the CeBIT fair in 2012 (see Fig. 1b).

The current version of Blinker, produced by BrainTech Ltd. [22], consists of 1920 white backlight diodes assembled into 320 controllable segments highlighting a $15^{\prime \prime}$ screen. Segments can be assigned to arbitrary-defined groups, which then act as one controllable stimulation field. The fields can have any size or shape, provided the chosen form can be assembled from the segments. Each field's light intensity has independently controlled waveform, phase, brightness, and frequency. The high brightness update rate $(5 \mathrm{kHz})$ of the diodes allows precise control of frequency (steps of $0.01 \mathrm{~Hz}$ ) and the stimulation phase. The backlight control board is connected via standard USB-C cable to a PC running Python-based drivers and control software. Reaction delay to the driving commands is kept below $0.5 \mathrm{~ms}$. The LCD screen itself is connected to a PC using a standard HDMI connection, allowing Blinker to present arbitrary content, applications, and media overlaid on the locally controllable, flashing backlight.

\subsection{Synchronization with EEG}

Internal clocks of the PC, an EEG amplifier, and Blinker, run independently. Classic synchronization based on signal sample number and the sampling frequency is not sufficient in this case - the clocks drift apart up to tens, or sometimes hundreds, of milliseconds per hour.

Blinker provides two methods of precise synchronization. The first one relies on the dedicated hardware synchronization output. It is a galvanically isolated output that can be set to repeat any of the flashing fields' signals. This signal can be recorded by an EEG-amplifier and used in subsequent signal processing routines. Additionally, Blinker's driver provides the time-stamp of stimulation onset in the PC-clock time. The time-stamp's generation algorithm compensates for the communication delays of the USB connection. An appropriate recording software, e.g., Svarog [23] or appropriate amplifier drivers or SDK, provide communicationdelay-corrected time-stamps for amplifiers samples as an additional signal channel. This channel can be used to synchronize stimulation events' onset with EEG signals. Results of the tests of synchronization precision are provided in Table II.

They are estimated on 1000 stimulations during an hour-long experiment. The average time lag was measured using a photodiode taped to one of the blinking fields. The photodiode was connected to the AUX input of an EEG amplifier, sampling at $F s=2048 \mathrm{~Hz}$. When using the synchronization cable, the average lag and its standard deviation can
TABLE II

Results of synchronization precision measurement.

\begin{tabular}{l|c|c}
\hline \hline $\begin{array}{c}\text { Synchronization } \\
\text { type }\end{array}$ & $\begin{array}{c}\text { Synchro } \\
\text { cable }\end{array}$ & $\begin{array}{c}\text { Event } \\
\text { time-stamps }\end{array}$ \\
\hline average lag [ms] & 0.08 & 0.38 \\
lag std. dev. [ms] & 0.19 & 0.61 \\
phase std. dev. at $20 \mathrm{~Hz}\left[{ }^{\circ}\right]$ & 1.37 & 4.39 \\
phase std. dev. at $40 \mathrm{~Hz}\left[{ }^{\circ}\right]$ & 2.74 & 8.78
\end{tabular}

be observed which is due to the fact that detection of the photodiode's signal rise is based upon crossing a threshold, and the signal is sampled at a finite frequency $F s$.

\section{Experimental study}

The presented experiment is an extension of our previous study [21] which was performed on a smaller group of users. Forty-nine healthy young adults (28 females and 21 males) participated in calibration and communication sessions of a high frequency SSVEP-BCI.

The study was approved by the Rectors Committee for Ethics of Research with Human Participants at the University of Warsaw, and informed consent was obtained from the volunteers.

\subsection{Procedure}

During the calibration, a participant was asked to concentrate on one of the fields, highlighted by different frequencies and labelled "yes" and "no", or on the field highlighted by steady light. The calibration was performed in short blocks. A brief auditory and visual instructions, indicating which blinking field the user should focus on, were presented before every block. Frequency of the stimulation was taken from the $25-40 \mathrm{~Hz}$ range. After every two blocks, the system validated the performance of the current frequency set based on the area under the ROC curve (AUC). AUC can be used for the assessment of valid detections [24]. Calibration and selection of an appropriate pair of frequencies continued until the system found a set of frequencies which provided AUC $\geq 0.8$.

The communication session started directly after the calibration. Participants' task was to answer twenty trivial questions (like, e.g., "Is the grass green?") via a binary YES/NO interface (see Fig. 1c). In each trial, the participant listened to the question displayed on the screen and then focused on the button corresponding to the correct answer. One second after the auditory presentation of the question, the response fields started flickering with the frequencies selected in the calibration, and continued until the classifier returned an answer. Then, the flickering stopped, and visual feedback was presented. 


\subsection{Data analysis}

In order to present results on a significant number of users, we included the data collected from the previous study [21]. This implied using the same experimental setup, so we also used the same processing pipelines. Their detailed description can be found in [21] and we also briefly recall them below.

Online causal filtering was applied to EEG signal using bandpass, second-order Butterworth filter with cut-off frequencies of 20 and $60 \mathrm{~Hz}$, and IIR notch filters at 50,100,150, $200 \mathrm{~Hz}$. The filtered EEG signal was stored in a circular buffer for analysis. During calibration, the buffer returned $1 \mathrm{~s}$ long signals every second, and during communication, the buffer produced $1 \mathrm{~s}$ long fragments every $0.5 \mathrm{~s}$. These fragments were then sent to the classifier for training/validation (during calibration) and decision making (during communication sessions).

The Lasso model [25, 26] was used to detect SSVEP responses. A design matrix was created consisting of $1 \mathrm{~s}$ long sines and cosines with the following frequencies: $\frac{1}{2} f_{y}, f_{y}, 2 f_{y}, \frac{1}{2} f_{n}, f_{n}, 2 f_{n}$, where $f_{y}$ and $f_{n}$ are the stimulation frequencies selected in the calibration for highlighting the "yes" and "no" fields, respectively. Using this matrix and a fragment yielded from the EEG circular buffer, LASSO model ${ }^{\dagger 1}$ was fitted. The resulting Lasso coefficients indicate to what degree the template matrix components were present in the EEG signal fragment. When the highest value of one of the Lasso coefficients exceeds the threshold set during the calibration session ${ }^{\dagger 2}$ twice in a row, then we declare that the frequency associated with that component is effectively present in the analyzed EEG signal. The item highlighted using that frequency is selected, and visual feedback is shown.

Accuracy of the communication is commonly defined as

$$
\mathrm{ACC}=\frac{N_{\text {corr }}}{N} \times 100 \% .
$$

In our case, $N_{\text {corr }}$ - the number of correctly answered questions - assuming the subjects knew the correct answers to (trivial) questions, $N$ - the total number of questions (in this study $N=20$ ).

Criterion for a "successful communication via BCI" is unfortunately seldom defined in studies reporting this outcome. We decided to use the $p$-value $p_{\text {bin }}$ of one-sided binomial test on the amount of correct yes/no answers, assuming zero hypothesis of random answers, and set the threshold at

$$
p_{\text {bin }}<0.05 \text {. }
$$

\footnotetext{
${ }^{1}$ Implementation in sklearn library sklearn.linear_model.coordinate_descent.Lasso. [26], with parameters differing from default: alpha $=1$, max_iter=1000, warm_start = True, selection=cyclic, fit_intercept=False

$\dagger^{2}$ Absolute value threshold and relative threshold compared to second highest components coefficient.
}

\subsection{Comparing results obtained on small and large groups}

Due to the factors inherent to biomedical sciences, BCI systems are often tested on relatively small user groups. For a small group size $N$, results can be non-representative in predicting the performance of $(N+1)$-st user. For larger groups, the reported mean accuracies provide better estimates of potential ability to communicate of a new BCI user. To compensate for this fact, we propose the application of the Laplace smoothing [27]. In our case, $C C_{L}$ simulates a situation when a group of $N$ BCI users, for whom results have been reported, is extended by two new users: one successful and one who fails. Hence,

$$
C C_{L}=\frac{C C+1}{N+2} \times 100 \%,
$$

where $C C$ is the number of successes (i.e., the reported participants who can communicate using high-frequency BCI-SSVEP), and $N$ is the total size of the tested group. This correction compensates for the high success rates, which can be achieved for small cohort sizes. Still, it does not significantly change the conclusions of studies performed on a significant number of subjects.

Figure 2 presents the reported mean accuracies after this smoothing (3).

\section{Results}

In the presented experiment, $93.9 \%$ of users (46 out of 49) were able to communicate successfully (2) via high-frequency BCI implemented on Blinker (Sect. 2), and their mean accuracy was 94.8\%. Specifically, 32 volunteers exceeded 95\%, 39 exceeded $90 \%$, and 43 exceeded $85 \%$ accuracy.

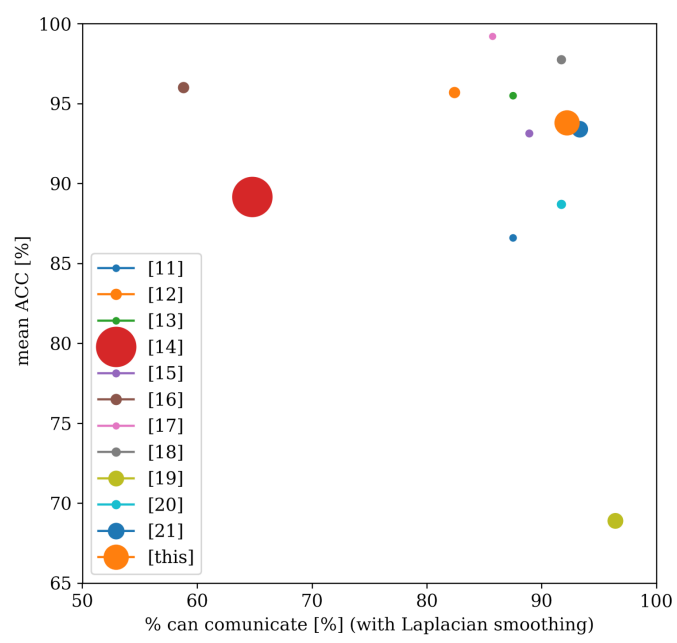

Fig. 2. The reported accuracy of high-frequency SSVEP-BCI operating online and our results relative to the percentage of users who were able to communicate. The percentage is shown using the Laplacian smoothing. The area of the marker dot represents the size of the tested group. 
To provide a reference for the efficiency of the proposed approach, we reviewed the available literature on high-frequency SSVEP-BCIs operating online. The best results reported in a given paper are presented in Table II and also in Fig. 2.

Each dot in Fig. 2 represents a given study from those collected in Table II. The vertical axis shows reported accuracies. In turn, the horizontal axis shows reported percentages of users, who were able to achieve successful communication via BCI, corrected by the Laplace smoothing (3). In spite of this smoothing, studies performed on small groups are still far less significant than studies on large groups. To visualize the relative sample sizes, each dot has the area proportional to the sample size of the corresponding study (labelled in the inset of Fig. 2). Such presentation allows for a quick assessment of the reported results in the three-dimensional space of the reported accuracies, success rates and sample sizes. We observe that only one study on highfrequency SSVEP-BCI was performed on a larger group, giving results inferior to the currently presented. Some studies [12, 13, 16-18] reported higher mean accuracies, but corresponding sample sizes were at least three times smaller, which decreases their relative significance. One study [19] reported a larger - even after the Laplacian smoothing percent of successfully communicating users with the lowest mean accuracy which sheds doubt on the significance of the reported accuracy (originally $100 \%$ ).

A typical effect of increasing the number of participants is represented by the difference between the currently presented results and our previous study [20] performed in the same experimental setup. Increasing the number of participants from 28 to 49 induced a drop in the success rate larger than estimated with (3).

\section{Summary}

We presented a novel, flexible and precise system for rendering stimuli for SSVEPs, which opens new possibilities in several fields. However, for its first verification we chose a standard application, which is the basic communication via highfrequency SSVEP-BCI. It showed superior performance in the context of comparable results, reported in the literature - even without taking into account the fact that most of these studies should be treated like proofs of concept, rather than tests of an actual proposition for a working BCI, applicable in real-world conditions.

Most of the contemporary high frequency SSVEP stimulators implement an ATM-like setup, with a fixed amount of LEDs surrounding a screen, or a fixed set of static buttons with hardware backlight. Blinker allows for a dynamic content presentation with just-in-place, flexible local backlight stimuli of an arbitrary shape. It allows users to focus their attention on the stimuli themselves, instead of refocusing the attention from the stimuli to the associated LED. Only such unobtrusive highlighting of items presented on the screen provides the comfort and ease of use of a BCI, required by the target groups of paralyzed or locked-in patients.

Among the technical advantages we should also recall the high precision of synchronization, achieved via duplication of the stimulation signal in the EEG recording or event timing synchronization in software, and the possibility of using arbitrary waveforms for modulating the intensity of the SSVEP-inducing backlight, like e.g., sines or square waves with arbitrary duty cycles and precisely controlled frequency and phase.

Finally, an unexplored advantage of the proposed approach lies the possibility of dynamical highlighting an almost arbitrarily chosen area of the screen with selected frequencies. It opens new opportunities in dynamic control of the phenomenon of selective attention: we can mark any objects or parts of an image, website, advertisement, or even a movie, with a specific frequency, and investigate which of these objects attract most of our covert and overt attention [3].

Combined, all these features open new possibilities in psychophysiological research, where SSVEP is used as a very sensitive indicator of attention, cognitive control and working memory mechanisms. Using almost invisible high frequency stimuli makes these procedures more natural and unobtrusive.

\section{References}

[1] F.-B. Vialatte, M. Maurice, J. Dauwels, A. Cichocki, Prog. Neurobiol. 90, 418 (2010).

[2] M.J. Wieser, A. Keil, J. Neurosci. 31, 7784 (2011).

[3] S. Walter, C. Quigley, S.K. Andersen, M.M. Mueller, Neurosci. Lett. 519, 37 (2012).

[4] S. Mun, M.-C. Park, S. Park, M. Whang, Neurosci. Lett. 525, 89 (2012).

[5] C.H. Attar, S.K. Andersen, M.M. Müller, Neuroimage 53, 1326 (2010).

[6] M.M. Müller, S. Hillyard, Clin. Neurophysiol. 111, 1544 (2000).

[7] Y. Kashiwase, K. Matsumiya, I. Kuriki, S. Shioiri, PLOS ONE 8, e70922 (2013).

[8] C.S. Herrmann, Exp. Brain Res. 137, 346 (2001).

[9] R.S. Fisher, G. Harding, G. Erba, G.L. Barkley, A. Wilkins, Epilepsia 46, 1426 (2005).

[10] P. Durka, R. Kuś, J. Żygierewicz, M. Michalska, P. Milanowski et al, Bull. Pol. Acad. Sci. Tech. Sci. 60, 427 (2012). 
[11] P.F. Diez, V.A. Mut, E.M.A. Perona, E.L. Leber, J. Neuroeng. Rehabilitat. 8, 39 (2011).

[12] P.F. Diez, S.M.T. Müller, V.A. Mut, E. Laciar, E. Avila, T.F. Bastos-Filho, M. Sarcinelli-Filho, Med. Eng. Phys. 35, 1155 (2013).

[13] D. Zhu, G. Garcia-Molina, V. Mihajlović, R.M. Aarts, in: Int. Conf. on Universal Access in Human-Computer Interaction, Springer, 2011, p. 645.

[14] I. Volosyak, D. Valbuena, T. Luth, T. Malechka, A. Graser, IEEE Trans. Neural Syst. Rehabilitat. Eng. 19, 232(2011).

[15] P.-L. Lee, J.-J. Sie, Y.-J. Liu, C.-H. Wu, M.-H. Lee, C.-H. Shu, P.-H. Li, C.-W. Sun, K.-K. Shyu, Ann. Biomed. Eng. 38, 2383 (2010).

[16] A. Chabuda, P. Durka, J. Żygierewicz, IEEE Trans. Neural Syst. Rehabilitat. Eng. 26, 344 (2017).

[17] S. Ajami, A. Mahnam, V. Abootalebi, Biocybern. Biomed. Eng. 38, 106 (2018).

[18] X. Chen, B. Zhao, Y. Wang, X. Gao, J. Neural Eng. 16, 026012 (2019).
[19] D.-O. Won, H.-J. Hwang, S. Dähne, K.-R. Müller, S.-W. Lee, J. Neural Eng. 13, 016014 (2015).

[20] Xiaogang Chen, Zhikai Chen, Shangkai Gao, Xiaorong Gao, Brain-Computer Interfaces 1, 181 (2014).

[21] A. Chabuda, M. Dovgialo, A. Duszyk, A. Stróż, M. Pawlisz, P. Durka, Acta Neurobiol. Exp. 79, 421 (2019).

[22] BrainTech, Blinker hardware.

[23] BrainTech, SVAROG, 2012.

[24] M. Dovgialo, A. Chabuda, A. Duszyk, M. Zieleniewska, M. Pietrzak, P. Różański, P. Durka, Int. J. Neural Syst. 29, 1850048 (2019).

[25] J. Friedman, T. Hastie, R. Tibshirani, J. Stat. Softw. 33, 1 (2010).

[26] F. Pedregosa, G. Varoquaux, A. Gramfort, V. Michel, B. Thirion et al., J. Mach. Learn. Res. 12, 2825 (2011).

[27] A. Agresti, B.A. Coull, Am. Stat. 52, 119 (1998). 